\title{
Multi-Modal Characterization of New Battery Technologies by Operando ec-STEM
}

B. L. Mehdi ${ }^{1,2}$, J. Chen ${ }^{1,3}$, A. Stevens ${ }^{4}$, C. Park ${ }^{5}$, L. Kovarik ${ }^{6}$, A. V. Liyu ${ }^{6}$, W. A. Henderson ${ }^{1}$, J-G. Zhang ${ }^{1,3}$, K. T. Mueller ${ }^{1,2}$, N. D. Browning ${ }^{2,7}$

${ }^{1}$ Joint Center for Energy Storage Research (JCESR), PNNL, Richland, WA, USA

${ }^{2}$ Physical and Computational Science, PNNL, Richland, WA, USA

${ }^{3}$ Energy and Environmental Directorate, PNNL, Richland, WA 99352, USA

${ }^{4}$ National Security Directorate, PNNL, Richland, WA, 99352, USA

${ }^{5}$ Industrial and Manufacturing Engineering, Florida State University, Tallahassee, FL 32306, USA

${ }^{6}$ EMSL, PNNL, Richland, WA, 99352, USA

${ }^{7}$ Materials Science and Engineering, University of Washington, Seattle, WA 99194, USA

The new generation energy storage materials such as Li-ion, Li-Sulfur, Li-Air etc. has generated the need for novel experimental techniques that provide real-time information on the dynamic structural changes and processes occurring locally at the electrode/electrolyte interface during battery operation (in operando). One of the most promising means to increase the energy density for example in case of state-of-the-art lithium Li-ion batteries is to replace the graphite anode with a Li metal anode. While the direct use of Li metal has many advantageous, at present issues related to dendrite growth and low Coulombic efficiency, CE, limit its practical application. Additionally, existing theory/models fall short of predicting emergent behaviors of heterogeneous electrochemical interfaces such as electrodeelectrolyte interfaces. This knowledge gap impedes the ability to design and control optimal interfaces for energy storage applications, which can lead to breakthrough discoveries in the energy storage field.

Here operando electrochemical scanning transmission electron microscopy (STEM) [1, 2] is used to directly image the deposition/stripping of $\mathrm{Li}$ at the anode/electrolyte interface in a Li-S battery. Lithium sulfur batteries are currently one of the most attractive battery systems for high capacity energy storage applications, potentially meeting a large demand by giving 3-5 fold higher energy densities (from 2600 Wh kg-1 to $2800 \mathrm{Wh} \mathrm{kg}^{-1}$ ) than state-of-the-art Li-ion batteries [3]. However, the practical application is still challenging due to the insulating nature of the elemental sulfur cathode, dissolution of intermediate discharge products (polysulfides, $\mathrm{Li}_{2} \mathrm{~S}_{\mathrm{n}}, 3 \leq \mathrm{n} \leq 6$ ) into the electrolyte, the "shuttle effect" and the formation of a passivating Solid Electrolyte Interphase (SEI) layer at the Li anode contributing to selfdischarge [4]. The multi-step electrochemical reduction and oxidation reactions involving polysulfides are very complex and require an increased fundamental understanding to bring Li-S technology to widespread commercialization. There are many strategies to improve the interfacial stability of the Li anode and control/suppress Li dendrite growth, which is highly dependent on nature of electrolyte itself, such as mixture of different electrolyte solvents, salts and additives. Here we demonstrate the changes in the Li dendrite growth mechanism in a Li-S battery in the presence of lithium nitride $\left(\mathrm{LiNO}_{3}\right)$ additive characterized by the multi-modal approach of in-situ TEM with compressive sensing $[5,6]$ and operando electrochemistry, XPS and NMR. As an example of the results obtained, Figure 1 shows a comparison of two different $\mathrm{Li}$ dendrite growth mechanism in the presence/absence of the additive in a commercially available Li-sulfur electrolyte. These results indicate that the mechanism is strictly related to the changes in coordination chemistry of Li in the two electrolyte solutions. In this presentation the origin and details of this reaction mechanism will be discussed [7]. 


\section{References:}

[1] B. L. Mehdi, J. Qian, E. Nasybulin, C. Park, D. A. Welch, R. Faller, H. Mehta, W. A. Henderson, W. Xu, C. M.Wang, J. E. Evans, J. -G. Zhang, K. T. Mueller, N. D. Browning, Nano Lett., 15, (2015), 2168

[2] B. L. Mehdi, A. Stevens, J. Qian, C. Park, W. Xu, W. A. Henderson, J-G. Zhang, K. T. Mueller, N. D. Browning, Sci. Rep., 6, (2016), 34267

[3] X. Ji, K. T. Lee, L. F. Nazar, Nat. Mater., 8, (2009), 500-506

[4] P. G. Bruce, S. A. Freunberger, L. J. Hardwick, J. M. Tarascon, Nat. Mater., 11, (2012), 19-29

[5] A. Stevens, H. Yang. L. Carin, I. Arslan and N. D. Browning, Microscopy 63, (2014) 41-51

[6] L. Kovarik, A. Stevens, A. Liyu, N. D. Browning, Applied Physics Letters 109, (2016) 164102

[7] This work was primarily supported by JCESR, an Energy Innovation Hub funded by DOE-BES. The development of the operando stage was supported by the Chemical Imaging LDRD Initiative at PNNL. PNNL is a multi-program national laboratory operated by Battelle for the U.S. DOE under Contract DE-AC05-76RL01830. A portion of the research was performed at the EMSL user facility sponsored by DOE-BER and located at PNNL. The multi-target tracking algorithm is supported by NSF-1334012.
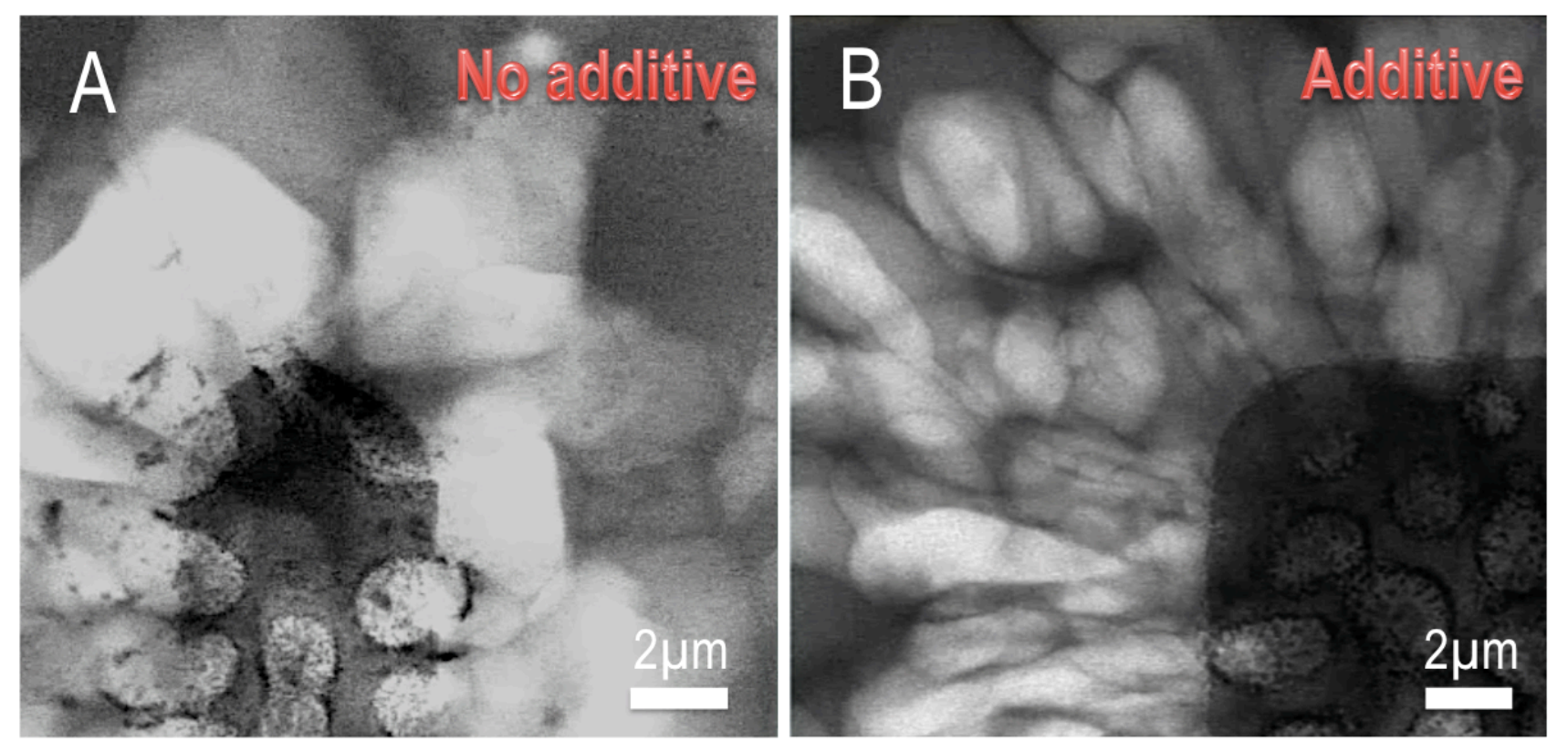

Figure 1. BF STEM images of Li dendrite deposits from Li-based electrolyte in (A) the absence of additive and (B) presence of additive showing the difference in dendrite growth mechanism. These phenomena demonstrate direct correlation between the heterogeneous dendrite growth and chemical composition of battery electrolyte as parameters controlling degradation process of Li-S battery. 